\title{
Live surgery broadcast: who is benefiting?
}

\author{
Frederik G. Dikkers ${ }^{1} \cdot$ J. Peter Klussmann ${ }^{2} \cdot$ Manuel Bernal-Sprekelsen $^{3}$ • \\ Cezary Mazurek $^{4} \cdot$ Witold Szyfter ${ }^{5}$
}

Received: 30 December 2015/Accepted: 8 March 2016/Published online: 17 March 2016

(C) Springer-Verlag Berlin Heidelberg 2016

All otorhinolaryngologists in academic centers are familiar with the difficulties involved regarding schooling, training and development of surgical skills in medicine, particularly in the complex field of otorhinolaryngology. However, several years ago, thanks to the Internet and its versatility as an educational tool, otology was successful in presenting and disseminating its knowledge by initiating live surgery broadcasts (LION). In 2015 a live surgery session was organized for rhinology (LION-ESS). Now it was time for Laryngology/Head and Neck Surgery. December 2, 2015, was the day the first European Laryngological Live Surgery Broadcast took place.

Participating surgeons came from eight hospitals in seven countries (Table 1). The organizers selected participants by approaching all members of the Scientific Council of the European Laryngological Society (ELS). All positive responders were included. Viewing of the broadcast was free of charge. In the organization of the broadcast, the potential audience was addressed by announcing

Frederik G. Dikkers

f.g.dikkers@umcg.nl

1 Department of Otorhinolaryngology, Head and Neck Surgery, University of Groningen, University Medical Center Groningen, P.O. Box 30.001, 9700 RB Groningen, The Netherlands

2 Department of Otorhinolaryngology, Head and Neck Surgery, University of Giessen, Giessen, Germany

3 Department of Otorhinolaryngology, Hospital Clinic, University of Barcelona Medical School, Barcelona, Spain

4 Poznań Supercomputing and Networking Center, Poznań, Poland

5 Department of Otolaryngology and Laryngological Oncology, University of Medical Sciences, Poznań, Poland the event to all members of the ELS, and by emailing all over the world making use of address lists of the sponsors. In addition, participants and ELS members were encouraged to contact address lists of their countries scientific societies.

All together, 15 live interventions were performed in three parallel sessions. These interventions were moderated from Poznań Supercomputing and Networking Center (PSNC) in Poznan, Poland, where four moderators were following all surgeries. PSNC is the operator of PIONIER-Research and Education Network in Poland. Its main activity profile is focused on research and development in Information and Communication Technologies (ICT).

For many years, scientific innovation has been a driving force of a real progress in the world. At the same time, scientific research, which is at the forefront of products and technologies available on the market and ensures proof of concept for new ideas, requires an advanced ICT infrastructure (e-infrastructure). Similarly, the progress in medicine requires integration with ICT methods and resources. Over 300 scientists and engineers from PSNC have already participated in over 100 international projects for many years. Here, original technologies, methods and applications are developed and deployed in collaboration, and for scientific community worldwide. Medicine can greatly benefit from this collaboration. For the first European Laryngological Live Surgery Broadcast scientists could use High Definition Scientific Television PlatonTV and medical teleconsultations platform medVC. These modalities were both ensuring high definition quality, multicamera and multisession broadcast for all. The moderators had audible live connections with the surgeons, enhancing visibility of relevant parts of the respective interventions. Moderators were further commenting on important and 
Table 1 Participating surgeons, hospitals and locations, alphabetically by country

\author{
Prof. A. Giovanni (Marseille University Hospital Conception, Marseille, France) \\ Prof. S. Lang (University Hospital Essen, Essen, Germany) \\ Prof. G. Peretti (San Martino University Hospital, Genoa, Italy) \\ Prof. M. Remacle (Centre Hospitalier de Luxembourg, Luxembourg, Luxembourg) \\ Dr. E. Sjögren, Dr. T. Langeveld (Leiden University Medical Center, Leiden, The Netherlands) \\ Prof. M. Wierzbicka (University of Medical Sciences, Poznań, Poland) \\ Prof. M. Quer (Hospital Sant Pau i Santa Creu, Barcelona, Spain) \\ Prof. I. Vilaseca (Hospital Clinic, University of Barcelona Medical School, Barcelona, Spain)
}

critical steps of the surgery and putting questions to the surgeons to help in clarifying procedures. At the end of the session, a discussion was conducted. All surgeons were present in their respective surgical theaters, ready to answer questions of the audience. The audience was encouraged to send questions to an email address that was repeatedly displayed.

Ultimately, some 1000 computers had logged in from all over Europe, but also from places as far as Pakistan, South Africa, Lebanon, the USA and Argentina. The organization received confirmation of the fact that some otorhinolaryngologic departments closed their ongoing work to allow their co-workers to join this session. We are aware of a broadcast in a lecture hall, to show the interventions to medical students.

During broadcast, 89 questions were received from all over the world. The subjects of the questions are displayed in Table 2. It is interesting to note that phonosurgery was the topic of the majority of questions. They concerned both surgical treatment details as well as assessment of vocal cords function after larynx surgery in short and in longterm follow-up. The most relevant were summarized and discussed. This was highly interesting, as different surgeons had opposite opinions. This added to the intellectual level of the replies. Obviously, the audience benefited of the broadcast.

What about the patients? Of course, patients had accepted through a written consent to participate in the session. However, there are several potential drawbacks. The surgeon might be at a higher stress level impairing his work, knowing that there is a critical audience pending on his/her surgical steps. Theoretically, the audience might even have included the patient's relatives, as this was an open broadcast. Also, the patient is able to review his own intervention afterward, as the session, including all complete fifteen surgical interventions, can now be searched on the Internet (http://els.livesurgery.net/). Potentially, an intervention might have been postponed, because a surgeon might have thought that this particular case would do so well during that broadcast. Despite the educational benefits of a live surgery broadcast, significant ethical concerns about patient's safety, clinical integrity and pressure on the surgeon still remain. There is conflicting evidence in the literature regarding the influence of live transmission related stress on surgeon performance [1-3]. Remarkably enough, the first institutional use of live broadcast surgeries of patients with severe proliferative diabetic retinopathy by closed circuit TV to their families in the hospital in the early nineties of the previous century has fostered great communication and confidence among patients, their families, and doctors [4]. Additionally, it has been shown that students viewing a live surgery learned significantly more and definitely were more positive about the live teleconferencing than students viewing only a video-tape [5]. In the first European Laryngological Live Surgery Broadcast, surgeons performed complex surgical procedures at the surgeon's home institute, to eliminate the issue surrounding an unfamiliar environment and to minimize risk and reduce stress.

Surgeons were all well aware of the fact that the organizers stressed that their particular intervention was not exclusively relevant to the success of the broadcast, having three parallel sessions. The surgery performed online was exactly the one performed in case the surgery had not been

Table 2 Most common questions $(n=89)$ asked during live surgery broadcast

\begin{tabular}{ll}
\hline Subject & Approximate percentage of all questions \\
\hline External thyroplasty & 60 \\
Endoscopic treatment of the laryngeal precancerous lesions and cancers & 15 \\
Open horizontal partial laryngectomy & 15 \\
Other & 10 \\
\hline
\end{tabular}


broadcasted. The advantage here was our possibility to switch to another setting and let the surgeon know that we were doing so in order to avoid pressure on him/her. This is not what usually happens during live performances with just one operating theater. This way, the organization was not only ready to easily switch between channels in case of a technical issue, but also in case of a medical emergency.

All together, it is the opinion of the organization that live broadcast of laryngologic surgery is feasible and attractive too, where the benefits for education outweigh the potential drawbacks for the consented patient. This new media offers an easy participation and an immediate interactive feedback of the users. Therefore, the ELS is seriously considering to organize the second European Laryngological Live Surgery Broadcast.

\section{Compliance with ethical standards}

Conflict of interest All authors declare that they have no conflicts of interest.

Ethical approval This article does not contain any studies with human participants or animals performed by any of the authors.
Informed consent Informed consent was obtained from all individual participants included in the study.

\section{References}

1. Khan SA, Chang RT, Ahmed K, Knoll T, van Velthoven R, Challacombe B et al (2014) Live surgical education: a perspective from the surgeons who perform it. BJU Int 114(1):151-158

2. Philip-Watson J, Khan SA, Hadjipavlou M, Rane A, Knoll T (2014) Live surgery at conferences — clinical benefits and ethical dilemmas. Arab J Urol 12(3):183-186

3. Williams JB, Mathews R, D'Amico TA (2011) "Reality surgery"-a research ethics perspective on the live broadcast of surgical procedures. J Surg Educ 68(1):58-61

4. Yoshida A (1998) The importance of informed consent in the field of ophthalmology (article in Japanese). Hokkaido Igaku Zasshi 73(1):15-20

5. Park A, Schwartz RW, Witzke DB, Roth JS, Mastrangelo M, Birch DW et al (2001) A pilot study of new approaches to teaching anatomy and pathology. Surg Endosc 15(3):245-250 